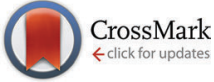

Cite this: Phys. Chem. Chem. Phys., $2016,18,32125$

Received 7th September 2016, Accepted 3rd November 2016

DOI: $10.1039 / c 6 c p 06175 c$

www.rsc.org/pccp

\title{
Conductivity and phase morphology of carbon black-filled immiscible polymer blends under creep: an experimental and theoretical study
}

\begin{abstract}
Yamin Pan, ${ }^{a}$ Xianhu Liu, ${ }^{a}{ }^{a}$ Xiaoqiong Hao ${ }^{b}$ and Dirk W. Schubert*a
Blends of carbon black (CB)-filled co-continuous immiscible polystyrene/poly(methyl-methacrylate) (PS/PMMA) with a PS/PMMA ratio of $50 / 50$ and CB selectively located in the PS phase have been prepared by melt blending. The simultaneous evolution of conductivity and phase morphology of blend composites was investigated under shear and in the quiescent state at $200{ }^{\circ} \mathrm{C}$. It was found that shear deformation had a significant influence on the conductivity of the unfilled PS/PMMA blend and its composites, which was attributed to the change of phase morphology during shear. After the shear stress of $10 \mathrm{kPa}$, the conductivity of PS/PMMA blends filled with 2 vol\% of CB decreased by about two orders of magnitude and the phase morphology transformed from a fine co-continuous structure into a highly elongated lamellar structure. The deformation of phase morphology and the decrease of conductivity were weakened upon decreasing the shear stress or increasing the $C B$ concentration. During subsequent recovery, pronounced phase structure coarsening was observed in the mixture and the conductivity increased as well. A simple model describing the behavior of conductivity under shear deformation was derived and utilized for the description of the experimental data. For the first time, the Burgers model was used to describe the conductivity, and the viscoelastic and viscoplastic parameters were deduced by fitting the conductivity under shear. The results obtained in this study provide a deeper insight into the evolution of phase structure in the conductive polymer blend composite induced by shear deformation.
\end{abstract}

\section{Introduction}

Conductive polymer composites have attracted significant interest due to their wide range of applications and ease of fabrication..$^{1-3}$ Conductive fillers such as carbon black (CB), carbon nanotubes and graphene are incorporated into various polymers to fabricate conductive polymer composites. However, the conductive fillers in polymer composites may increase the viscosity of composites and thus lead to poor processability. ${ }^{1-14} \mathrm{~A}$ low percolation threshold is therefore desired. The design of the double percolated structure is an effective way of lowering the percolation threshold. In the double percolated structure, conductive fillers are added into the immiscible polymer blend and preferably distributed in one continuous phase or selectively localized at the interface of the polymer blend. ${ }^{4-14}$ In our previous work, ${ }^{7}$ a low percolation threshold was obtained for polystyrene/poly(methyl-methacrylate) (PS/PMMA)/CB composites by applying the double percolation

\footnotetext{
${ }^{a}$ Institute of Polymer Materials, Friedrich-Alexander University Erlangen-Nuremberg, Martensstr. 7, 91058 Erlangen, Germany. E-mail: xianhu.liu@fau.de, dirk.schubert@fau.de

${ }^{b}$ School of Science, Xi'an Jiaotong University, Xi'an, Shaanxi, 710049, China
}

concept with CB located in the PS phase. The addition of high CB concentration efficiently suppressed the structure coarsening during further annealing treatment.

The understanding and control of the blend composite morphology development in mixing equipment where two or more immiscible polymers are melt blended is a long-lasting dream of researchers in the field of polymer processing. ${ }^{15-21}$ The structure of conductive pathways and the phase morphology in the final material are significantly influenced by the shear or elongation forces applied during processing. ${ }^{22-29}$ Therefore, understanding the effects of a defined flow field on composite structures and their phase morphology evolution is vital for the preparation of conductive polymer composites with desired electrical properties. ${ }^{30-35}$ However, the deformation behaviour of the blend composite under pure shear or elongational flow is still not fully understood.

To date, most of these studies are only focused on conductive polymer composites with a single polymer as the matrix material. $^{22-29}$ To our knowledge, the simultaneous electrical and rheological measurement as a tool to reveal the relationship between shear deformation and conductive networks in polymer blend composites has been rarely reported, and in particular, 
there are no publications available on a corresponding change of phase morphology. In the present work, the simultaneous electrical and rheological experiments were performed on PS/PMMA/CB composites. Besides that, scanning electron microscopy was utilized to characterize the development of phase morphology in the blend composites during creep and recovery processes. Moreover, a simple model was postulated to describe the conductivity of the polymer blend composite under shear. From these experiments it is expected to achieve a deeper insight into the changes of the phase morphology during shear deformation and their impact on conductivity.

\section{Experimental}

\section{Materials}

Commercial PMMA Plexiglas 6N (density $1.19 \mathrm{~g} \mathrm{~cm}^{-3}$; mass average molar mass $M_{\mathrm{W}}=85.2 \mathrm{~kg} \mathrm{~mol}^{-1}$ ) and PS $158 \mathrm{~N}$ (density $1.05 \mathrm{~g} \mathrm{~cm}^{-3} ; M_{\mathrm{W}}=268 \mathrm{~kg} \mathrm{~mol}{ }^{-1}$ ) were provided by Evonik Röhm GmbH, Germany and Styrolution Group GmbH, Germany, respectively. CB Printex XE2 was provided by Evonik with a specific surface area of $900 \mathrm{~m}^{2} \mathrm{~g}^{-1}$ measured using the BET-method. The mean diameter of the primary CB particles was around $35 \mathrm{~nm}$ and the density at room temperature was $2.13 \mathrm{~g} \mathrm{~cm}^{-3}$.

\section{Sample preparation}

All materials were dried at $80{ }^{\circ} \mathrm{C}$ in a vacuum oven for at least $18 \mathrm{~h}$ prior to processing. The PS/PMMA (50/50 vol\%) blends filled with different CB concentrations from 0 to 6 vol\% were prepared by melt mixing at $200{ }^{\circ} \mathrm{C}$ (Haake polyDrive 557-8310, Thermo Scientific, Germany). Both polymers and CB were introduced directly into the mixer at a rotation speed of $20 \mathrm{rpm}$. After $2 \mathrm{~min}$ of mixing at $20 \mathrm{rpm}$, the rotational speed was increased to $60 \mathrm{rpm}$ for $8 \mathrm{~min}$. After the melt mixing, the materials were quenched to room temperature and then granulated using a blade granulator (Wanner Technik, Germany). The obtained granulates were compression molded in a hot press (Voigt, Germany) into $2 \mathrm{~mm}$ thick disks with a diameter of $25 \mathrm{~mm}$. They were first preheated at $200{ }^{\circ} \mathrm{C}$ for 5 min under vacuum; and then pressed at 100 bar for 2 min before being quenched at room temperature for $10 \mathrm{~min}$.

\section{Characterization}

The phase morphology of the compression-molded samples was examined using scanning electron microscopy (SEM, Carl Zeiss Microscopy, Germany) with an accelerating voltage of $3 \mathrm{kV}$. The molded specimens were fractured in liquid nitrogen to obtain undeformed fracture surfaces and the surfaces were then coated with gold using a Sputter Coater S150B from Edwards, UK. To obtain a better contrast, the PMMA phase of some samples was etched away before coating according to the following procedure. An ultramicrotome U2 from Reichert with a glass knife was used to obtain a smooth surface at room temperature and the PMMA phase was then selectively etched with glacial acetic acid at $60{ }^{\circ} \mathrm{C}$ for 1 hour; after which the etched specimens were washed with ethanol in an ultrasonic bath for $40 \mathrm{~min}$.

In order to prepare samples with a shear history, a stresscontrolled Gemini shear rheometer from Malvern Instruments with a Picoammeter 6487 from Keithley was used. ${ }^{29}$ After inserting the specimen between the plates of the rheometer and reaching the measuring temperature of $200{ }^{\circ} \mathrm{C}$, the sample was kept for $10 \mathrm{~min}$ under quiescent conditions prior to shear. Then a constant shear stress ( 1 to $10 \mathrm{kPa}$ ) was applied for $60 \mathrm{~s}$. To observe the electrical behavior of composites during the recovery period the conductivity was recorded further after the shear step. In order to conserve the morphology of blend composites, the samples were quenched to room temperature immediately after the corresponding step using a cooling spray based on tetrafluoroethane (Kontakt Chemie; Kälte 75). The conductivity $\sigma$ was calculated by

$$
\sigma=d / R \pi r^{2}
$$

where $R, d$ and $r$ are the resistance, thickness and radius of the sample, respectively. Every simultaneous measurement was performed at least three times on three individual specimens. The experimental curves presented are representative examples of the data obtained.

\section{Results and discussion}

\section{Phase morphology}

Fig. 1a-d display the SEM images, respectively, for the unfilled PS/PMMA blend and its composites with various CB loadings after selective etching of the PMMA phase (the CB distribution in the PS/PMMA/CB composites was examined by transmission electron microscopy and it was found that the CB agglomerates were selectively located in the PS phase ${ }^{7}$ ). Clearly, all samples showed a typical co-continuous morphology. With increasing $\mathrm{CB}$ concentration, the phase structure became finer. A refinement of the blend morphology after the addition of solid particles is usually explained by an interfacial activity of the filler particles and/or by a change in the rheological behaviour of the components during mixing. In the system, the studied CB is localized inside

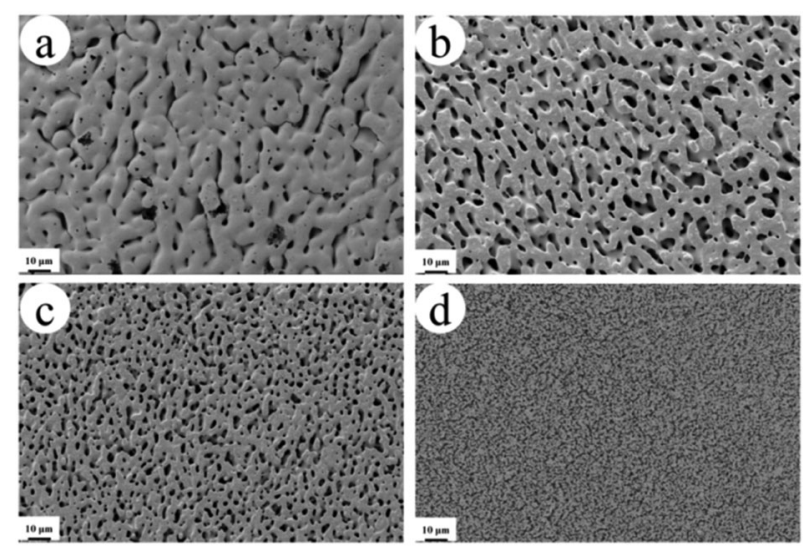

Fig. 1 SEM images of PS/PMMA/CB composites: SEM images of blends without (a) and with (b) 1 vol\%, (c) 2 vol\% and (d) 4 vol\% CB. 


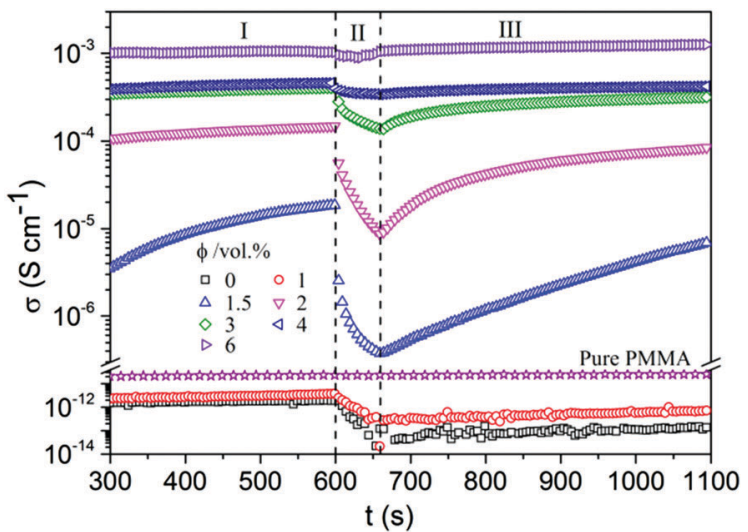

Fig. 2 Typical example of conductivity evolution for PS/PMMA/CB composites with various $\mathrm{CB}$ contents during electrical-rheological experiments at $200{ }^{\circ} \mathrm{C}$ involving a shear step $\left(\tau_{\mathrm{c}}=5 \mathrm{kPa}, t_{\mathrm{c}}=60 \mathrm{~s}\right)$.

the PS only and, thus, lowering the interfacial tension or immobilization of the interface can hardly explain distinctly the finer phase structure of filled blends. Therefore, morphology refinement could be explained by the increased PS phase viscosity filled with $\mathrm{CB}$, thus retarding the phase-coarsening process.

\section{Conductivity of PS/PMMA/CB composites under shear}

Fig. 2 shows the representative resulting conductivity evolution of pure PMMA and PS/PMMA/CB composites with various $\mathrm{CB}$ loadings under shear and quiescent conditions at $200{ }^{\circ} \mathrm{C}$ (the conductivity of pure PS is lower than our detection limit). In region I the conductivity was monitored under quiescent conditions for $600 \mathrm{~s}$. In region II a constant stress $\tau_{\mathrm{c}}$ of $5 \mathrm{kPa}$ with a creep time $t_{\mathrm{c}}$ of $60 \mathrm{~s}$ was applied and the conductivity was measured simultaneously. After the shear, the conductivity measurements were continued in order to study the recovery behavior (region III). Concerning the initial conductivity values one can determine the region of the percolation threshold. The unfilled PS/PMMA blend and the composite with 1 vol\% of CB are nonconductive materials. Upon increasing the $\mathrm{CB}$ content to $1.5 \mathrm{vol} \%$, a tremendous increase in conductivity of about six orders of magnitude can be observed. A further increase in the filler amount leads to higher conductivity values; however, the increase is not so pronounced anymore. This indicates that the percolation threshold is around 1.5 vol\% of CB.

During the first $600 \mathrm{~s}$ of annealing under quiescent conditions (region I), no change in the conductivity can be observed for the pure PMMA, the unfilled PS/PMMA blend and the composite with 1 vol\% CB. However, an obvious increase in the conductivity about one order of magnitude was found for the composite with 1.5 vol\% CB, which should be attributed to the dynamic percolation and changes in the phase morphology. Firstly, for composites containing nanofillers, the thermal motion of the particles in the melt and attractive forces between them lead to the formation of conductive paths under quiescent conditions, i.e. dynamic percolation. ${ }^{36-40}$ Secondly, the phase morphology of polymer blend composites is usually in a non-equilibrium state. In this case a reduction in the interfacial area and an increase in the size of the phase domains often occur during further processing or in the molten state, thus altering the space arrangement of the filler and, hence, the conductivity properties. When the CB concentration was further increased, the conductivity increased slightly due to the formation of more dense and more stable CB conductive pathways. ${ }^{23,28}$

During the shear step (region II), it was interesting to find that the polymer blending and CB concentration had a significant influence on the composites' conductivity. For a pure PMMA matrix, there was no change in the conductivity occurring during the shear stage. However, for the unfilled PS/PMMA blend and its composite with 1 vol\% of $\mathrm{CB}$, the conductivity values dropped about one order of magnitude after a shear stress of $5 \mathrm{kPa}$. The reason for this phenomenon was attributed to the change of phase morphology during shear. For the PS/PMMA/CB composite with 1.5 vol\% CB, the conductivity of the composite dropped about two orders of magnitude after the shear stress of $5 \mathrm{kPa}$. With a further increase of the CB concentration, the particle structures and the phase morphology were more stable, as more particles were involved in the conductive pathways, and the conductivity drop became less significant. However, due to the change of phase morphology during the shear stage, the conductivity variation was still visible at the high CB content ( 6 vol\%), which was more obvious than that of the single polymer composite systems with the same concentration. ${ }^{23}$ Removing the shear stress (region III), a recovery of the conductivity was observed due to reorganization of the conductive network.

To quantify the relative conductivity drop, a parameter $\sigma_{\mathrm{D}}=\left(\sigma_{0}-\sigma_{\min }\right) / \sigma_{0}$ was introduced, where $\sigma_{\min }$ is the minimum conductivity reached during shear and $\sigma_{0}$ is the conductivity exactly before the shear stress. Fig. 3 shows the relative conductivity drop $\sigma_{\mathrm{D}}$ as a function of the CB content. Due to the co-continuous structure, one considered the specimen as a parallel circuit of resistors representing individual PMMA or PS phases. For the unfilled blend, one neglected the conductivity of the PS matrix because the PMMA matrix $\left(10^{-11} \mathrm{~S} \mathrm{~cm}^{-1}\right)$ had a higher conductivity than the PS matrix $\left(10^{-16} \mathrm{~S} \mathrm{~cm}^{-1}\right)$. For $\mathrm{CB}$ filled composites,

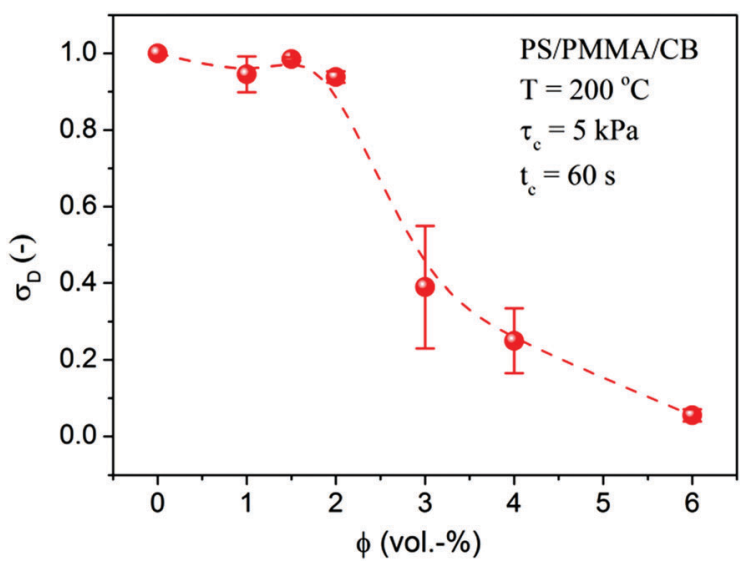

Fig. 3 The relative conductivity drop induced by the creep deformation $\left(T=200{ }^{\circ} \mathrm{C}, \tau_{\mathrm{c}}=5 \mathrm{kPa}, t_{\mathrm{c}}=60 \mathrm{~s}\right)$ as a function of $\mathrm{CB}$ concentration. 
one could neglect the conductivity of the PMMA matrix because the CB particles were located in the PS phase. ${ }^{7}$ At CB concentration below the percolation threshold, there were no conductive pathways in the material. But the morphology of PMMA and PS phases was significantly affected by the shear, thus leading to a dramatical decrease of the conductivity. At $\mathrm{CB}$ concentrations above the percolation threshold, especially around the percolation threshold (1.5 vol\%), a distinct destruction of particle structures induced by a creep step at $5 \mathrm{kPa}$ was observed. However, upon increasing the CB concentration, the stability of the particle structures and the phase morphology was increased; therefore, the relative conductivity drop diminished, which is consistent with the single polymer composites. ${ }^{23}$

Tests were also performed to compare the conductivity of blend composites at different stress levels. Here, due to the initial conductivity values of the blend composite span, the conductivity values were normalized by the last value before the shear stress was applied for the purpose of lucidity. Fig. 4 shows a typical example of normalized conductivities of PS/PMMA/CB composites under different creep stresses. Clearly, the high stress level can bring a large conductivity drop. The relative conductivity drop $\sigma_{\mathrm{D}}$ was extracted from the experimental data as shown in Fig. 5. It was obvious that upon increasing the shear stress to a critical value, such as $5 \mathrm{kPa}$ for the blend composites with 1.5 and $2 \mathrm{vol} \% \mathrm{CB}$, the relative conductivity
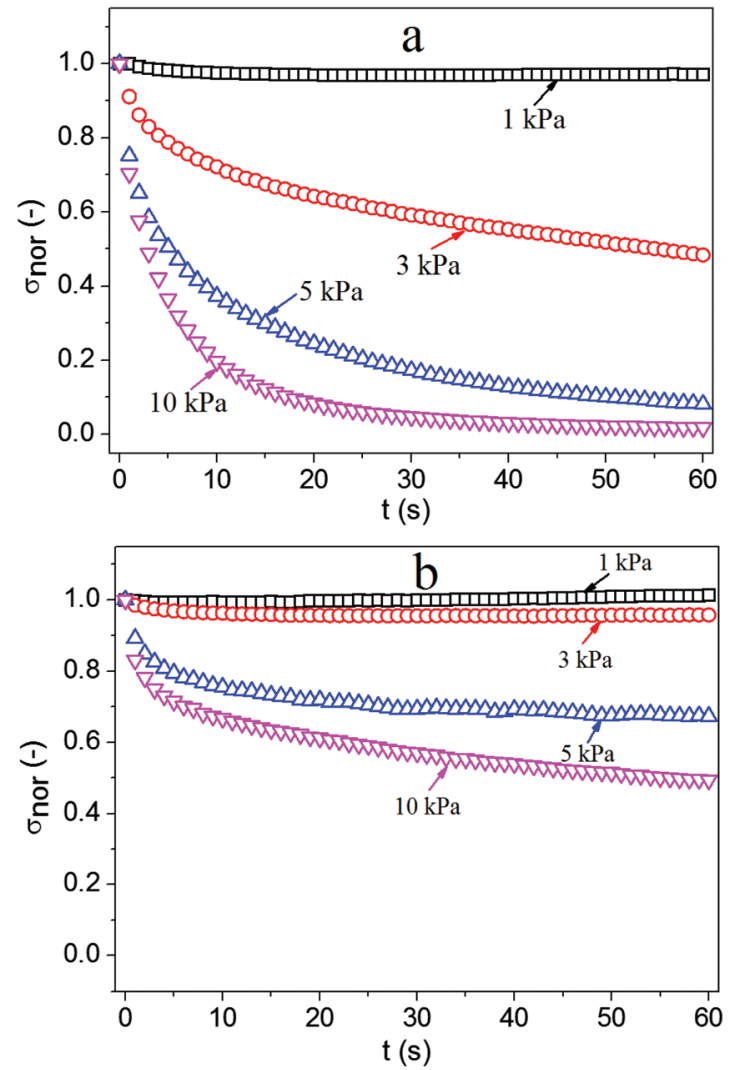

Fig. 4 Typical example of normalized conductivities of PS/PMMA/CB composites with (a) 2 vol\% and (b) 4 vol\% CB under different creep stresses $\left(T=200{ }^{\circ} \mathrm{C}, t_{\mathrm{C}}=60 \mathrm{~s}\right)$.

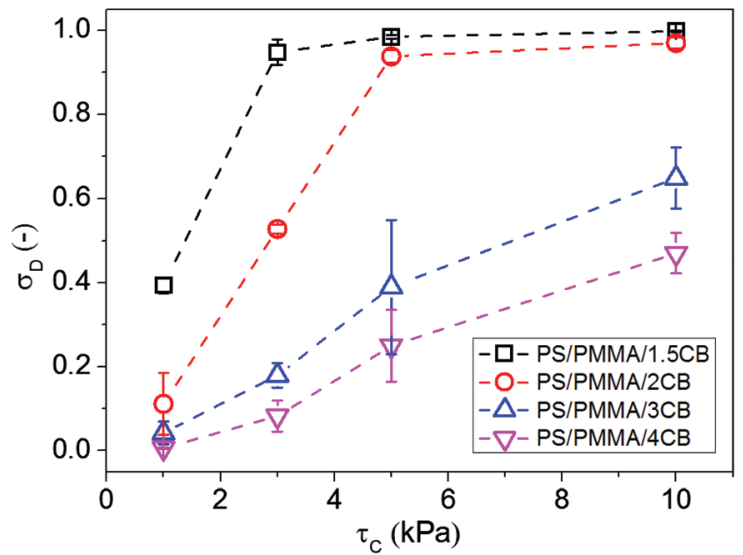

Fig. 5 The relative conductivity drop as a function of creep stress $\left(T=200^{\circ} \mathrm{C}\right.$, $\left.t_{c}=60 \mathrm{~s}\right)$ for PS/PMMA/CB composites with different CB concentrations.

drop of all composites increased steeply. This behavior became less pronounced as the $\mathrm{CB}$ concentration was further increased. This maybe because the higher loading of CB into the PS phase causes an increase in the viscosity of the PS phase. ${ }^{5}$ Therefore, the composites with higher $\mathrm{CB}$ loading are more resistant to phase morphology changes induced by shear deformation.

\section{Phase morphology of PS/PMMA/CB composites under shear}

In order to better understand the change of the phase morphology of PS/PMMA/CB composites under shear deformation, Fig. 6 shows a typical example of SEM images describing the morphological evolution of the PS/PMMA/CB composite with 2 vol\% CB along the time axis. At the initial point "a", a fine co-continuous structure was observed. After annealing at $200{ }^{\circ} \mathrm{C}$ for $600 \mathrm{~s}$ (Point b), the composite sample underwent phase morphology coarsening due to the minimization of interfacial free energy ${ }^{41-44}$ After the shear for $60 \mathrm{~s}$ (Point c), the domains were significantly deformed and appeared as elongated lamellar structures, indicating that the phase morphology deformed significantly after the application of the large shear. During the

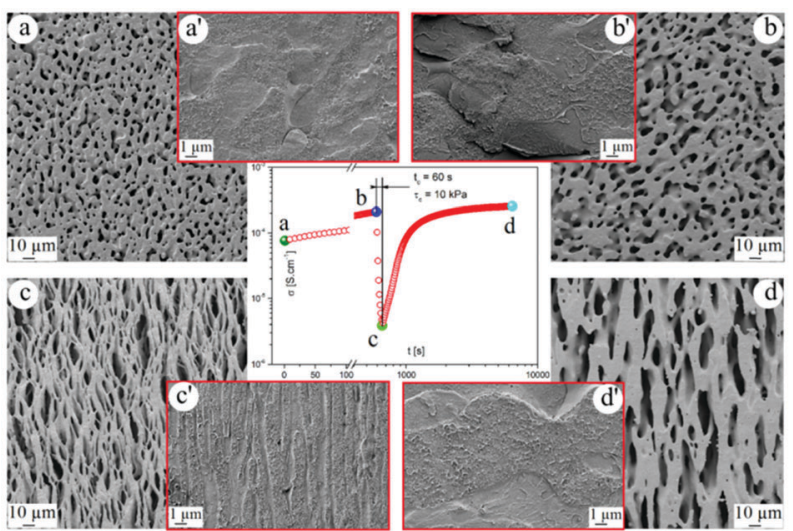

Fig. 6 SEM images of PS/PMMA/CB composites with 2 vol\% CB involving a shear step $\left(\tau_{\mathrm{c}}=10 \mathrm{kPa}, t_{\mathrm{c}}=60 \mathrm{~s}\right)$ : (a and $\left.\mathrm{a}^{\prime}\right)$ initial state, $\left(\mathrm{b}\right.$ and $\left.\mathrm{b}^{\prime}\right)$ after annealing $(600 \mathrm{~s}),\left(c\right.$ and $\left.c^{\prime}\right)$ after creep shear (60 s), and ( $d$ and $\left.d^{\prime}\right)$ after recovery (6000 s). 


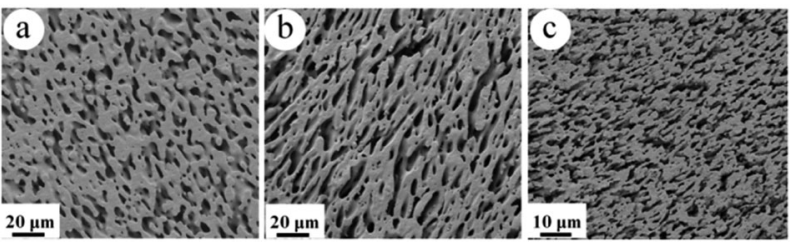

Fig. 7 SEM images of PS/PMMA/CB composites ( $a$ and b: 2 vol\% CB; $\mathrm{C}$ : 4 vol\% $\mathrm{CB})$ after shear $\left(T=200{ }^{\circ} \mathrm{C}, t_{\mathrm{C}}=60 \mathrm{~s}\right)$ with different shear stresses: (a) $1 \mathrm{kPa}$, (b) $5 \mathrm{kPa}$ and (c) $10 \mathrm{kPa}$.

recovery period the phase morphology changed from a highly elongated lamellar structure to a fine-range co-continuous structure (Point d). These results indicated that the application of shear significantly changed the phase morphology and the conductivity of PS/PMMA/CB composites. Additionally, the impact of shear on the phase morphology became less significant, as the CB concentration was increased or the shear stress decreased, as shown in Fig. 7, which is in good agreement with the conductivity results in Fig. 5.

\section{Model}

Motivated by the existing idea and experimental findings we proposed a simple model to describe the conductivity drop behavior during shear deformation. ${ }^{22,27}$ The scenario was schematically presented by a model as shown in Fig. 8. Due to the co-continuous structure, in Fig. 8 we have idealized a lamellar-phase in the specimen. We have ignored the phase in the horizontal direction because the electrical conductivity was tested along the perpendicular direction. In Fig. 8a we considered the lamellarphase without shear with length $l_{0}$ and cross section area $A_{0}$. With the addition of $\mathrm{CB}$, the lamellar-phase became slim and its number increased (Fig. 8b, this effect was confirmed by the SEM images in Fig. 1). However, for the unfilled blend or composites

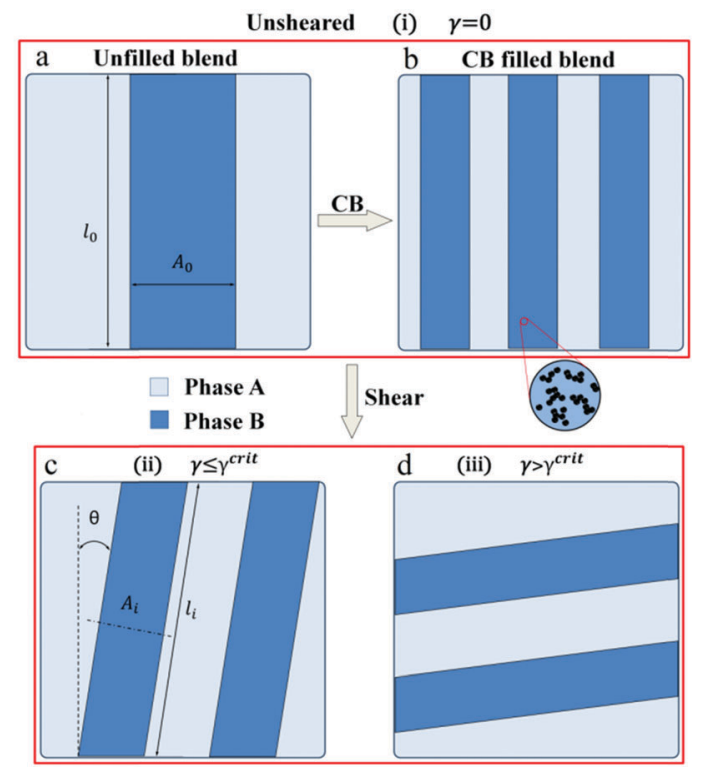

Fig. 8 Sketch of a model for the phase morphology of the polymer blend and its composite under different shear states. The shear direction is horizontal direction. with the same CB concentration, we assumed that each lamellar-phase in the blend had the same length $l_{0}$ and cross section area $A_{0}$. At first, we considered those lamellar-phases only under very small deformations (state ii, Fig. 8c). Therefore, under shear the lamellar-phase was stretched resulting in an increase in the length $l_{\mathrm{i}}$ and a decrease in the area $A_{\mathrm{i}}$ perpendicular to the axis of the sheared lamellar-phase. Under the assumptions that the volume of the lamellar-phase remained constant under shear (eqn (2)) and that the conductive pathways remain intact during shear, which means only a rearrangement of the $\mathrm{CB}$ aggregates takes place. Thus, one can write

$$
A_{0} l_{0}=A_{\mathrm{i}} l_{\mathrm{i}}=V
$$

Therefore, the electrical resistance of the elongated lamellarphase is defined as

$$
R_{\mathrm{i}}\left(l_{\mathrm{i}}\right)=\rho l_{\mathrm{i}}^{2} / V
$$

where $\rho$ represents the electrical resistivity of the polymer matrix or the CB filled polymer matrix. In our case, for the unfilled blend, $\rho$ represents the electrical resistivity of the PMMA matrix, and for CB filled composites, $\rho$ represents the electrical resistivity of the PS/CB phase. The length of the sheared lamellar-phase was furthermore defined as

$$
l_{\mathrm{i}}(\theta)=l_{0} / \cos (\theta)
$$

where $\theta$ is the deformation angle. Then eqn (3) becomes

$$
R_{\mathrm{i}}(\theta)=\rho\left[l_{0} / \cos (\theta)\right]^{2} / V
$$

The deformation $\gamma$ was defined as $\gamma=\tan \theta$ and reduced for deformation angles smaller than 0.5 rad to $\gamma \approx \theta$. Moreover, the conductivity is proportional to the resistance $\sigma \propto 1 / R$. Accordingly, eqn (5) becomes

$$
\sigma(t)=A[\cos (\gamma)]^{2}
$$

where $A=V / \rho l_{0}{ }^{2}$. For the purpose of lucidity, the conductivity values were normalized. Therefore, eqn (6) is rewritten in the following forms:

$$
\sigma_{\text {nor }}(t)=\left[\cos (\gamma)^{2}\right]
$$

or

$$
\gamma(t)=\arccos \sqrt{\sigma_{\text {nor }}(t)}
$$

The assumption of our model was only applied to an appropriate shear deformation. Under a large shear deformation as shown in Fig. 8d, the parallel lamellar-phase along the thickness direction in the polymer blend can be rearranged along the shear direction and the conductive pathways would be destroyed. And under a small deformation, the conductivity and the phase morphology were not affected by shear as shown in Fig. 5 and 7 .

The Burgers model is a combination of Maxwell and KelvinVoigt elements, as shown in Fig. 9a. According to the Burgers model, the total deformation is the sum of three essentially separate parts: ${ }^{45}$

$$
\gamma(t)=\gamma_{\mathrm{E}}+\gamma_{\mathrm{V} 1}+\gamma_{\mathrm{VP}}
$$


where subscripts E, V1 and VP correspond to the elastic, viscoelastic and viscoplastic contributions. However, the instantaneous deformation $\gamma_{E}$ in the molten state is very small. Therefore, the Burgers model presented here is simplified as shown in Fig. 9b. Accordingly, the time dependence of $\gamma(t)$ can be written as

$$
\gamma(t)=\frac{\tau_{\mathrm{c}}}{E_{\mathrm{V} 1}}\left[1-\mathrm{e}^{-t / \lambda}\right]+\frac{\tau_{\mathrm{c}}}{\eta_{\mathrm{VP}}} t
$$

where $\tau_{\mathrm{c}}$ is the creep stress, $E_{\mathrm{V} 1}$ is the elastic moduli, $\lambda=\frac{\eta_{\mathrm{V} 1}}{E_{\mathrm{V} 1}}$ is the retardation time, and $\eta_{\mathrm{VP}}$ is the viscosity. Thus, based on eqn (8) and (10), the viscoelastic and viscoplastic parameters can be obtained:

$$
y=\frac{\arccos \sqrt{\sigma_{\mathrm{nor}}(t)}}{\tau_{\mathrm{c}}}=\frac{1}{E_{\mathrm{V} 1}}\left[1-\mathrm{e}^{-t / \lambda}\right]+\frac{\tau}{\eta_{\mathrm{VP}}}
$$

Assuming that

$$
\begin{aligned}
\eta_{\mathrm{V} 1} & =\eta_{\mathrm{V} 10}{ }^{*} f(\varphi) \\
E_{\mathrm{V} 1} & =E_{\mathrm{V} 10}{ }^{*} f(\varphi)
\end{aligned}
$$

with

$$
f(\varphi)=1+a \varphi+b \varphi^{2}
$$

where $\eta_{\mathrm{V} 10}$ and $E_{\mathrm{V} 10}$ are the viscosity and elastic moduli for the unfilled PS/PMMA blend, respectively. And the CB concentration range is from 0 to 1 . This means that the concentration dependence $f(\varphi)$ of the sub-Kelvin model, $E_{\mathrm{V} 1}$ and $\eta_{\mathrm{v} 1}$, are equal, and then resulting in a concentration independent $\lambda$.

Fig. 10 shows the master curves of PS/PMMA/CB composites with different $\mathrm{CB}$ concentrations under appropriate shear stress. The solid lines correspond to the fit according to eqn (11). As expected, when the CB concentration is higher than the percolation threshold, the curves master very well for the composites within experimental accuracy for the same CB concentration under a certain shear stress region, which clearly demonstrates a linear material behaviour. The Burgers model description results are in good accordance with the experimental data for the

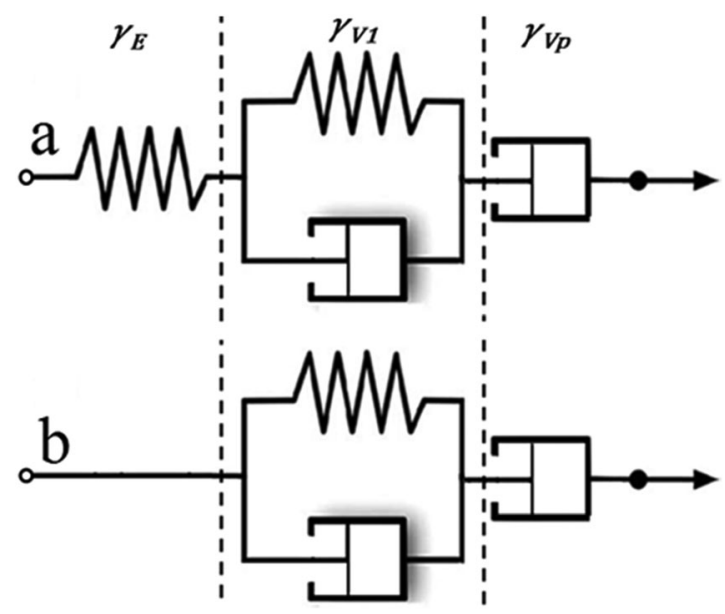

Fig. 9 Schematic representation of the Burgers model.

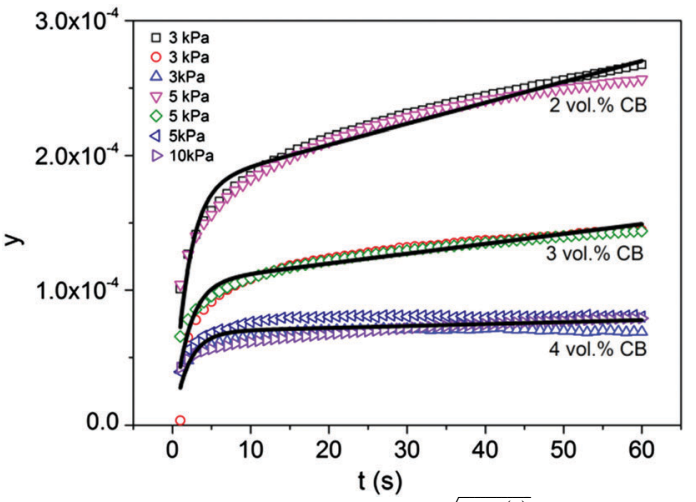

Fig. 10 Typical examples of $y=\frac{\arccos \sqrt{\sigma_{\text {nor }}(t)}}{\tau_{\mathrm{c}}}$ for PS/PMMA/CB composites under the appropriate shear stress: solid lines fitted by eqn (11).

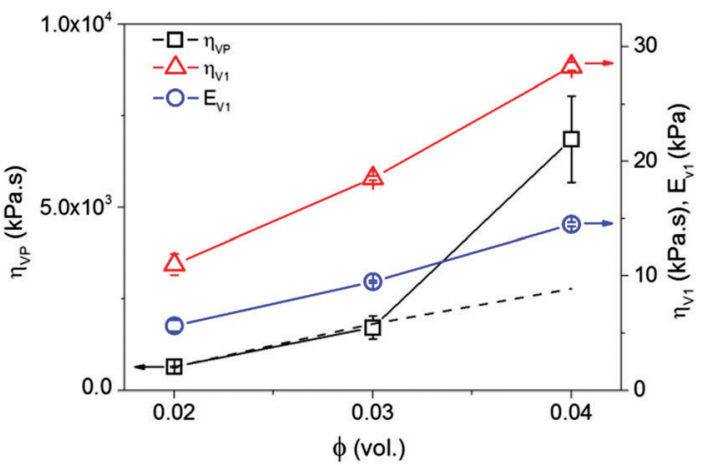

Fig. 11 Fitting parameters from eqn (11) with a constant value of $\lambda=1.95$ s. Dash line: by applying $f(\varphi)=1+74.81 \varphi+4222.22 \varphi^{2}$.

PS/PMMA/CB composites. The viscoelastic and viscoplastic parameters are shown in Fig. 11. As can be seen from Fig. 11, $\eta_{\mathrm{VP}}, \eta_{\mathrm{V} 1}$ and $E_{\mathrm{V} 1}$ are increased with increasing $\mathrm{CB}$ concentration, which indicates the reinforced mechanical properties of the blend composites and the increased resistance to viscous flow. ${ }^{46-48}$ Moreover, from the $E_{\mathrm{V} 1}$ data, the $f(\varphi)$ function was calculated as $f(\varphi)=1+74.81 \varphi+4222.22 \varphi^{2}$, which is in good agreement with $f(\varphi)=1+72.85 \varphi+4176.03 \varphi^{2}$ from $\eta_{\mathrm{V} 1}$.

For the CB concentration around the percolation threshold (1.5 vol\%) and for composites under the high shear stress (10 kPa), the curves cannot overlap for the composites, because our model is based on the assumption that the conductive pathways remain intact during shear. Nevertheless, for the first time, the Burgers model was used to describe the conductivity, and the viscoelastic and viscoplastic parameters based on the Burgers model were deduced by fitting the conductivity under shear.

\section{Conclusions}

The simultaneous evolution of the conductivity and phase morphology of co-continuous immiscible PS/PMMA/CB composites was investigated under shear and in the quiescent molten state. Under the shear stress of $10 \mathrm{kPa}$, the conductivity of the PS/PMMA (50/50) blend with 2 vol\% CB dropped about two orders of 
magnitude and the phase morphology changed into a highly elongated lamellar structure. The deformation of phase morphology and the decrease of conductivity were weakened upon decreasing the shear stress or increasing the CB concentration. During the subsequent recovery, pronounced phase structure coarsening and increased conductivity were observed in the composites. A simple model was derived and utilized for the description of the behavior of the conductivity drop under shear deformation. For the first time, the Burgers model was used to describe the conductivity, and the viscoelastic and viscoplastic parameters based on the Burgers model were deduced by fitting the conductivity under shear. The results obtained in this study provide a deeper insight into the evolution of phase structure in conductive polymer blend composites induced by shear deformation.

\section{Acknowledgements}

Y. Pan, X. Liu and X. Hao acknowledge the China Scholarship Council for funding the scholarship.

\section{Notes and references}

1 H. Deng, L. Lin, M. Ji, S. Zhang, M. Yang and Q. Fu, Prog. Polym. Sci., 2014, 39, 627-655.

2 H. Liu, J. Gao, W. Huang, K. Dai, G. Zheng, C. Liu, C. Shen, X. Yan, J. Guo and Z. Guo, Nanoscale, 2016, 8, 12977-12989.

3 X. Zhang, S. Zheng, X. Zheng, Z. Liu, W. Yang and M. Yang, Phys. Chem. Chem. Phys., 2016, 18, 8081-8087.

4 F. Gubbels, S. Blacher, E. Vanlathem, R. Jérôme, R. Deltour, F. Brouers and P. Teyssie, Macromolecules, 1995, 28, 1559-1566.

5 S. L. Scherzer, E. Pavlova, J. D. Esper and Z. Starý, Compos. Sci. Technol., 2015, 119, 138-147.

6 C. Zhang, X. S. Yi, H. Yui, S. Asai and M. Sumita, Mater. Lett., 1998, 36, 186-190.

7 Y. Pan, X. Liu, X. Hao, Z. Starý and D. W. Schubert, Eur. Polym. J., 2016, 78, 106-115.

8 E. Laredo, M. Grimau, A. Bello, D. F. Wu, Y. S. Zhang and D. P. Lin, Biomacromolecules, 2010, 11, 1339-1347.

9 C. Mao, Y. Zhu and W. Jiang, ACS Appl. Mater. Interfaces, 2012, 4, 5281-5286.

10 M. Liebscher, M. Blais, P. Pötschke and G. Heinrich, Polymer, 2013, 54, 5875-5882.

11 J. Huang, C. Mao, Y. Zhu, W. Jiang and X. Yang, Carbon, 2014, 73, 267-274.

12 S. Zhang, H. Deng, Q. Zhang and Q. Fu, ACS Appl. Mater. Interfaces, 2014, 6, 6835-6844.

13 G. Nasti, G. Gentile, P. Cerruti, C. Carfagna and V. Ambrogi, Polymer, 2016, 99, 193-203.

14 Y. Song, C. Xu and Q. Zheng, Soft Matter, 2014, 10, 2685-2692.

15 J. K. Lee and C. D. Han, Polymer, 1999, 40, 6277-6296.

16 J. K. Lee and C. D. Han, Polymer, 2000, 41, 1799-1815.

17 H. Li and U. Sundararaj, Macromol. Chem. Phys., 2009, 210, 852-863.

18 Z. Starý, F. Machui and H. Münstedt, Polymer, 2010, 51, 3744-3752.
19 G. Zheng, W. Yang, B. Yin, M. Yang, C. Liu and C. Shen, J. Appl. Polym. Sci., 2006, 102, 3069-3077.

20 K. Dai, X. B. Xu and Z. M. Li, Polymer, 2007, 48, 849-859.

21 C. Shen, Y. Zhou, R. Dou, W. Wang, B. Yin and M. Yang, Polymer, 2015, 56, 395-405.

22 X. Liu, J. Krückel, G. Zheng and D. W. Schubert, Compos. Sci. Technol., 2014, 100, 99-104.

23 Z. Starý, J. Krückel and D. W. Schubert, Polymer, 2014, 55, 3980-3986.

24 Z. Starý, Polymer, 2014, 55, 5608-5611.

25 X. Liu, J. Krückel, G. Zheng and D. W. Schubert, ACS Appl. Mater. Interfaces, 2013, 5, 8857-8860.

26 J. Krückel, Z. Starý and D. W. Schubert, Polymer, 2013, 54, 1106-1113.

27 J. Krückel and D. W. Schubert, Eur. Polym. J., 2014, 53, 50-57.

28 X. Liu, Y. Pan, G. Zheng and D. W. Schubert, Compos. Sci. Technol., 2016, 128, 1-7.

29 J. Krückel, Z. Starý, C. Triebel, D. W. Schubert and H. Münstedt, Polymer, 2012, 53, 395-402.

30 C. Mao, J. Huang, Y. Zhu, W. Jiang, Q. Tang and X. Ma, J. Phys. Chem. Lett., 2012, 4, 43-47.

31 F. Yu, H. Deng, Q. Zhang, K. Wang, C. Zhang, F. Chen and Q. Fu, Polymer, 2013, 54, 6425-6436.

32 J. Huang, Y. Zhu, W. Jiang, J. Yin, Q. Tang and X. Yang, ACS Appl. Mater. Interfaces, 2014, 6, 1754-1758.

33 J. Huang, Y. Zhu, W. Jiang and Q. Tang, Composites, Part A, 2015, 69, 240-246.

34 L. Su, P. Wang, Z. Xu, M. Peng and D. Chen, J. Polym. Sci., Part B: Polym. Phys., 2016, 54, 369-373.

35 J. Huang, J. Xu, Y. Sheng, Y. Zhu, W. Jiang, D. Xu, Q. Tang and X. Nie, Macromol. Mater. Eng., 2016, 301, 743-749.

36 C. Zhang, P. Wang, C. A. Ma, G. Wu and M. Sumita, Polymer, 2006, 47, 466-473.

37 H. Pang, C. Chen, Y. Zhang, P. Ren, D. Yan and Z. M. Li, Carbon, 2011, 49, 1980-1988.

38 W. Li, Y. Zhang, J. Yang, J. Zhang, Y. Niu and Z. G. Wang, ACS Appl. Mater. Interfaces, 2012, 4, 6468-6478.

39 Y. Zhang, D. Zheng, H. Pang, J. Tang and Z. M. Li, Compos. Sci. Technol., 2012, 72, 1875-1881.

40 X. Liu, Y. Pan, X. Hao, K. Dai and D. W. Schubert, J. Appl. Polym. Sci., 2016, 133, 43810.

41 V. Altstädt, L. L. de Freitas and D. W. Schubert, Pure Appl. Chem., 2004, 76, 389-413.

42 X. Cai, B. Li, Y. Pan and G. Wu, Polymer, 2012, 53, 259-266. 43 X. Liu, Z. Sun, R. Bao, W. Yang, B. Xie and M. Yang, RSC Adv., 2014, 4, 41059-41068.

44 X. Liu, R. Li, R. Bao, W. Jiang, W. Yang, B. Xie and M. Yang, Soft Matter, 2014, 10, 3587-3596.

45 I. M. Ward, Mechanical Properties of Solid Polymers, John Wiley \& Sons, 1983.

46 Y. Jia, K. Peng, X. Gong and Z. Zhang, Int. J. Plast., 2011, 27, 1239-1251.

47 Z. Yao, D. Wu, C. Chen and M. Zhang, Composites, Part A, 2013, 50, 65-72.

48 X. Wang, L. Gong, L. Tang, K. Peng, Y. Pei, L. Zhao, L. Wu and J. Jiang, Composites, Part A, 2015, 69, 288-298. 